\title{
sciendo
}

\section{Mini-Basketball Training Program Improves Physical Fitness and Social Communication in Preschool Children with Autism Spectrum Disorders}

\author{
by \\ Ke-Long Cai ${ }^{1}$, Jin-Gui Wang', Zhi-Mei Liu' ${ }^{1}$, Li-Na Zhu' ${ }^{2}$, Xuan Xiong ${ }^{1}$, \\ Sebastian Klich', Adam Maszczyk4, Ai-Guo Chen ${ }^{1,5}$
}

This investigation examined the effects of a 12-week mini-basketball training program (MBTP) on physical fitness and social communication in preschool children with autism spectrum disorders (ASD). The study applied a quasi-experimental design. Fifty-nine preschool children aged 3-6 years with ASD were assigned to either a MBTP group $(n=30)$ or a control group $(n=29)$. Participants in the MBTP group received a scheduled mini-basketball training program (5 sessions per week, forty minutes per session) for twelve consecutive weeks, while the control group was instructed to maintain their daily activities. The physical fitness test and the parent-reported Social Responsiveness Scale Second Edition (SRS-2) test were performed before and after the intervention. Results indicated that the 12-week MBTP facilitated performance in the physical fitness test, particularly in speed-agility and muscular strength abilities. Additionally, children in the MBTP group demonstrated improvement in SRS-2 performance in social awareness, social cognition, social communication, and autistic mannerisms, whereas no such changes were found in the control group. It may be concluded that the 12-week MBTP could improve physical fitness and social communication in preschool children with ASD, and thus the use of physical exercise intervention as a therapeutic tool for preschoolers with $A S D$ is recommended.

Key words: mini-basketball, physical fitness, social communication, preschool children, autism spectrum disorders, quasi-experimental.

\section{Introduction}

According to the USA Centers for Disease Control and Prevention (CDCP) in April 2018, the prevalence of Autism Spectrum Disorder (ASD) has raised from $1 / 5000$ in 1975 to $1 / 59$ (Baio et al., 2018). The increasing prevalence of ASD represents a major public health challenge. Impairment in social communication predominates among one of the core diagnostic features of ASD (American Psychiatric Association, 2013), including deficits in socialemotional reciprocity, non-verbal communicative behaviors, as well as developing, maintaining, and understanding relationships (Müller and Fishman, 2018). Notably, ASD children with social communication impairment may demonstrate lower physical activity levels, a more sedentary lifestyle, and much worse exercise tolerance compared to normal developing counterparts (Must et al., 2014; Pan and Frey, 2006). Bedford et al. (2016) have previously reported that early motor disabilities are often predictive of later social communication impairment in ASD. A previous study has revealed that physical

1 - College of Physical Education, Yangzhou University, Yangzhou, China.

2 - School of Physical Education and Sports Science, Beijing Normal University, Beijing, China.

3 - Department of Paralympic Sport, University School of Physical Education in Wroctaw, Wroctaw, Poland.

4 - Institute of Sport Sciences, Academy of Physical Education in Katowice, Katowice, Poland.

5 - Chinese - Polish Laboratory on Sport and Brain Science. 
fitnessdisability is associated with social communication impairment in children with ASD (Tyler et al., 2014). The association highlights the importance of rehabilitation in physical fitness and social communication for ASD (American Psychiatric Association, 2013). Consequently, a variety of behavioral and psychosocial treatments are sought by families, yet there is little consensus on which of them are most effective (Sharda et al., 2018).

Compared with other treatment methods, lower cost, no side effects, and easier administration have been recognized as advantages of physical exercise, which has become an alternative rehabilitation method for individuals with ASD. Previous reviews have been conducted regarding the impact of physical activity or exercise intervention on children and adults with ASD and all reported promising results (Bremer et al., 2016; Lang et al., 2010; Petrus et al., 2008; Sorensen and Zarrett, 2014; Sowa and Meulenbroek, 2012). Apart from improvements in physical fitness (Pitetti et al., 2007; Todd and Reid, 2006), improvements in social-communication have also been reported in children with ASD after swimming and aqua aerobics (Pan, 2010), horseback riding (Bass et al., 2009; Gabriels et al., 2012), Kata techniques training (Movahedi et al., 2013) and leisure activity programs (Garcia-Villamisar and Dattilo, 2011). However, to date, the majority of studies in the field of social-communication have recruited school-aged participants ( $>7$ years old) or adults with high-functioning autism for investigation. There have been few studies focusing on preschool children (3-6 years old) with ASD. Children with ASD have shown different social communication impairment at different stages of growth (Zhao and Fan, 2017). Preschool children, in particular, do not often engage in social settings, reducing opportunities for socialcommunicative development that can impair their quality of life and is of great concern to their caregivers. Therefore, there is a growing consensus that early intervention is extremely important and it will directly affect the prognosis of children with ASD (Wang and Wang, 2014).

To address these gaps in the area, the present study focused on preschool children with ASD. To the best of our knowledge, no studies on training programs dedicated to preschool children with ASD have been published. Thus, our research team consisting of experts from multiple disciplines, such as pediatrics, psychology, sport sciences, and physical education, designed the present mini-basketball training program (MBTP) to provide more comprehensive support for children with ASD. Since one of the core characteristics of ASD is social communication impairment, physical activity is often overlooked in this population. This study aimed to understand the effects of a 12-week MBTP on social communication and physical fitness in preschool children with ASD. The main hypothesis was that preschool children with ASD who participated in the 12-week MBTP would show improvements in physical fitness and social communication compared to children who did not receive the MBTP. The success of the study would provide evidence for an effective, inexpensive, easy-to-administer and relatively non-specialized intervention that may be scaled in varied settings across cultures, addressing the need for globally applicable intervention models for ASD in preschool-aged children.

\section{Methods}

\section{Participants}

Ninety-four children aged 3-6 years were recruited from the Yangzhou Chuying Child Development Center and Starssailor Education Institute (Yangzhou, P. R. China). Participants were excluded if: (1) they had participated in basketball training or regular physical exercise in the past 6 months; (2) had one or more co-morbid psychiatric disorders; (3) had a complex neurological disorder (e.g., epilepsy, phenylketonuria, fragile $X$ syndrome, tuberous sclerosis); (4) presented visual and auditory disorders; (5) had a medical history of head trauma; (6) presented any medical condition that might limit the exercise intervention.

Thirty-five children were excluded due to abovementioned reasons or declined to participate. Eligible participants from the Starssailor Education Institution were as assigned to the MBTP group $(n=30)$, while participants from the Chuying Child Development Center were chosen as a control group $(n=29)$. Finally, only thirty participants completed the entire experiment, while twenty-nine dropped out in the present study (Figure 1). 


\section{Study Design}

The study presents a quasi-experimental design, with the group defined as the betweensubject factor and the time point as the withinsubject factor. Eligible children were assigned either to a MBTP group or a control group. Before the intervention began, the detailed demographics of each child had been obtained. Additionally, parent-reported on SRS-2, CSHQ, CEBQ, and assessor-conducted physical fitness test outcomes were collected. To introduce the MBTP to all parents and children with ASD, a workshop was held.

This study was conducted between October and December 2018 in Yangzhou (P. R. China) with ethics approval from the Ethics and Human Protection Committee of the Affiliated Hospital of the Yangzhou University. This study has been registered in the Chinese Clinical Trial Registry (ChiCTR1900024973). Written informed consent was obtained from the participants' parents/guardians. The personal details of each child were not revealed in the present study; a videotape or photos of the training sessions will be used for teaching or presentation purposes, but the children's and their parents' faces were blurred on the videos/photos so as not to be recognized.

\section{Procedures}

All participants followed rehabilitation (ABA, Floor Time, RDI, TEACCH, etc.) in two institutions. On this basis, the MBTP group had an additional mini-basketball session, while the control group did not. The duration of the MBTP was 12 weeks in total. Each session was conducted by two professional physical education teachers in each institution. At least one parent of the participating children was required to join the class and accompany their child during the training session. The intervention was implemented as prescribed and supervised by the healthcare providers biweekly.

In the post-test, each participant completed the physical fitness test conducted by the same assessors in the same place. The participant's parent completed the SRS-2. All parent/legal guardian report measures were rated by a person consistent for each participant. Finally, participants and their parents/legal guardians received fair remuneration for their involvement in the experiment.
The mini-basketball training program (MBTP) was adapted from previous studies (Bagherly et al., 2012; Lambert et al., 2016; Zhu et al, 2017). The MBTP was mainly carried out in a collective class, which provided a lot of interaction and communication for children with ASD. Parents were encouraged to join the class and accompany their child during the session, in which children benefited from social interaction with their peers, teachers and were given the constant attention of their parents.

All of the training prescriptions were structured to achieve particular benefits that would be related to physical fitness and social communication. The MBTP comprised one session per day, five days per week for 12 consecutive weeks (60 sessions in total). Each session lasted 40 min and comprised the following four stages: (a) a 5 min warm-up; (b) 20 min basic basketball skill learning; (c) 10 min basketball game, and (d) a 5 min cool-down.

The MBTP was designed to include various levels of difficulty so that teachers could administer the session at appropriate levels for children. This might also motivate children to progress to higher levels in the course of the training program. The 12-week MBTP was divided into three phases to achieve different targets:

\section{a) Phase I (2 Weeks)}

To arouse children's interest in minibasketball, and standardize classroom routines in children and their parents, the main content was interesting and simple basketball training. At this stage children learned standard behavior and classroom routines such as taking turns, waiting and obeying.

\section{b) Phase II (8 Weeks)}

To improve children's mini-basketball skills and physical fitness, the main content was physical fitness training (shuttle running, leapfrogging, duck walking, etc.), basic basketball skills (dribbling, passing, shooting, etc.). Additionally, peer coordination training (passing and catching the ball, relay racing, etc.) was used to stimulate children's social communication behavior.

\section{c) Phase III (2 Weeks)}

To improve children's cooperative abilities, social skills, and collectivization, the main content was basketball group games such as a basketball- 
dribbling relay, basketball-passing relays, basketmoving shooting, etc.

The participants' demographics (age, gender, body mass and height, and BMI) were obtained at baseline (Table 1). The autism severity level of participants was confirmed using the Childhood Autism Rating Scale (CARS) (Schopler et al., 1980) and a detailed clinical assessment report was provided. Additionally, we found that social communication was associated with sleep disorders and eating behaviors in children with ASD. To ensure all participants' homogenization, sleep problems and eating behavior were taken into consideration as confounding variables and were evaluated at baseline. Sleep problems were assessed using the Children's Sleep Habits Questionnaire (CSHQ) (Owens et al., 2000), a parent-reported questionnaire designed as a screening instrument for sleep disorders. It has been validated for preschool children with ASD and has a high degree of correspondence (Goodlin-Jones et al., 2008). The children's eating style was assessed by a parent-report Child Eating Behavior Questionnaire (CEBQ) (Wardle et al., 2001).

Children's physical fitness was assessed by a China's manual of physical fitness (General Administration of Sport of China, 2003), which comprises the measurements of body mass and height to assess anthropometry, the $2 \times 10$ meter shuttle run test for speed-agility, standing long jump tests for muscular strength, the sit-and-reach test for flexibility and the balance beam test for balance. All participants completed the physical fitness test conducted by assessors in the autism institutes.

For the shuttle run, two parallel lines were drawn on the floor $10 \mathrm{~m}$ apart. Participants ran back and forth as fast as possible crossing each line with both feet every time. This test was performed twice, covering a total distance of $20 \mathrm{~m}$ $(2 \times 10 \mathrm{~m})$. The best of two attempts was recorded (s) for further analysis.

For the standing long jump, the participant stood behind the starting line, with feet together, pushed off vigorously and jumped forward as far as possible. The distance was measured from the take-off line to the point where the back of the heel nearest to the take-off line landed on the mat or non-slippery floor. The test was repeated three times, and the best score was retained $(\mathrm{cm})$.
During the sit-and-reach test, the participant sat on the floor with the legs held straight by an assessor, and bent forward slowly and reached as far as possible. There was a bench with the measurement scale in front of the participant whose hands were on the top of the bench, and the maximum reach was measured. The best result of the two attempts was recorded for further analysis $(\mathrm{cm})$.

For the balance beam test, participants stood on the starting point facing the balance beam (length $3 \mathrm{~m}$, width $10 \mathrm{~cm}$, height $30 \mathrm{~cm}$ ) with their arms flat on the side. When children heard the 'start' command, they moved forward. The tester started the stopwatch when children started and stopped it when they crossed the finish line. The test was repeated twice, and the best score was retained. If the score exceeded $30 \mathrm{~s}$ or the child was unable to complete the test, the score was recorded as $30 \mathrm{~s}$.

The Social Responsiveness Scale Second Edition (SRS-2) was used to measure changes in the severity of social communication impairment of participants in both groups (Constantino and Gruber, 2005). The SRS-2 was a 65-item rating scale that measured symptoms indicative of autism spectrum disorders. Each of the 65 items was rated on a 4-point frequency scale ranging from 1 (Not True) to 4 (Almost Always True). It was a parent-and/or teacher's reported measure of children's social impairment in naturalistic social settings that could be completed in $15 \mathrm{~min}$. Questions were the same in both the parent and teacher forms of the SRS-2. It generated scale scores for specific symptom domains (social awareness, social cognition, social communication, social motivation, and autistic mannerisms), as well as a total score that served as an index of severity of social impairment in ASD. Higher total scores on the SRS-2 indicated greater severity of social impairment. The SRS-2 manual reported internal consistency (Cronbach's alpha) for the total score for boys as 0.93 , and testretest reliability as 0.85 , with 17 months between testing. In the present study, parent-reported behavioral outcomes were shown on the SRS-2.

\section{Statistical Analysis}

Our sample size was determined using G*Power 3.1.9.2 (Erdfelder et al., 2009) and assumed a medium effect size (Cohen's $f=0.25$ ), power of 0.80 , alpha of 0.05 ; furthermore, analysis 
of repeated measures (ANOVA) with two groups (MBTP vs. control) was conducted. This indicated a sample of 24 (12 participants in each of the two groups). To control possible attrition over the two posttest time points, we increased this by $25 \%$, yielding the final sample of 30 used in this study.

A two-way mixed randomized controlled trial design was employed with group and time as independent variables. To ensure homogeneity in potential confounds between the MBTP and the control group, an analysis of independent samples using a $t$-test or a $\chi^{2}$-test was applied to compare demographic data between the two groups. Statistical analyses, testing the effect of the 12-week MBTP on physical fitness and social communication using the $2 \times 10 \mathrm{~m}$ shuttle run, standing long jump, sit-and-reach, balance beam test performances, and SRS-2 scores, were conducted separately using a 2 (group: MBTP vs. control) $\times 2$ (time: baseline vs. posttest) repeatedmeasures ANOVA, whereas the effect size was presented as partial eta-squared $\left(\eta^{2}\right)$ values. For the ANOVAs, significant interaction effects were followed up with tests of simple main effects. The data were presented as descriptive statistics: Mean \pm Standard Deviation $(\mathrm{M} \pm \mathrm{SD})$. An $\alpha$ of 0.05 was used as the level of statistical significance for all statistical analyses which were conducted using jamovi 1.0.7 (Retrieved from https://www.jamovi.org).

\section{Results}

Assessment at baseline consisted of detailed demographics and diagnostic reports (Table 1). Participants did not differ at baseline in age $[t(1,29)=.099, p>.05]$, gender $\left(\chi^{2}=.283, p>\right.$ $.05)$, BMI $[t(1,29)=.199, p>.05]$, severity (CARS) $[t(1,29)=.408, p>.05]$, sleep disorders (CSHQ) $[t$ $(1,29)=.559, p>.05]$ and eating behavior (CEBQ) $[t(1,29)=.704, p>.05]$ during the study. Mean and standard deviation of all children's physical fitness performances and the SRS-2 scores are shown in Table 2.

A two-factor analysis of variance of the $2 \times 10$ $\mathrm{m}$ shuttle run demonstrated a significant main effect of time $\left[F(1,28)=12.221, p<.05\right.$, partial $\eta^{2}=$ $.304]$ and an interaction of group by time $[F(1,28)$ $=5.532, p<.05$, partial $\left.\eta^{2}=.165\right]$. However, the posttest $2 \times 10 \mathrm{~m}$ shuttle run performance was faster than the baseline performance in the MBTP group $(p<.001)$, but not in the control group $(p>.05)$. For the standing long jump, a significant main effect of time $[F(1,28)=20.756, p<.001$, partial $\left.\eta^{2}=.426\right]$, group $[F(1,28)=4.384, p<.05$, partial $\left.\eta^{2}=.135\right]$ and an interaction of group by time $\left[F(1,28)=6.591, p<.05\right.$, partial $\left.\eta^{2}=.191\right]$ were found. A follow-up analysis deconstructing this interaction revealed no differences in baseline performance between the MBTP and control groups $(p>.05)$. However, the posttest standing long jump performances were significantly better compared to the baseline in the MBTP group $(p<$ $.001)$, but not in the control group $(p>.05)$ (Figure 2).

For the SRS-2 total score, there was no significant difference between the main effect of time $\left[F(1,28)=0.286, p>.05\right.$, partial $\left.\eta^{2}=.001\right]$ and group $\left[F(1,28)=0.001, p>.05\right.$, partial $\left.\eta^{2}=.000\right]$. However, there was a significant difference between the main effect of an interaction of group by the time $\left[F(1,28)=14.783, p<.001\right.$, partial $\eta^{2}=$. 346]. A follow-up analysis deconstructing this interaction revealed no differences in baseline scores between the MBTP and control groups $(p<$ .05). Nevertheless, the posttest SRS-2 total scores were lower than the baseline scores in the MBTP group $(p<.01)$, but higher than the baseline in the control group $(p<.05)$ (Figure 3). Similar findings were also observed for social awareness, social cognition, social communication, and autistic mannerisms, with no significant difference between the main effect of time and group $(p>$ .05). However, there was a significant difference between the main effect of an interaction of group by time $(p<.05)$. A follow-up analysis deconstructing all the interactions revealed no differences in baseline scores between the MBTP and control groups $(p>.05)$. The posttest social awareness $(p=0.062$, marginally significant $)$, social cognition $(p<.05)$ scores were lower than the baseline scores in the MBTP group, but not in the control group $(p>.05)$. The posttest social communication scores were lower compared to the baseline values in the MBTP group $(p<.01)$, but higher than the baseline in the control group $(p<.05)$. Additionally, the posttest autistic mannerisms scores were higher than the baseline scores in the control group $(p<.05)$, but not in the MBTP group $(p>.05)$ (Figure 4). 


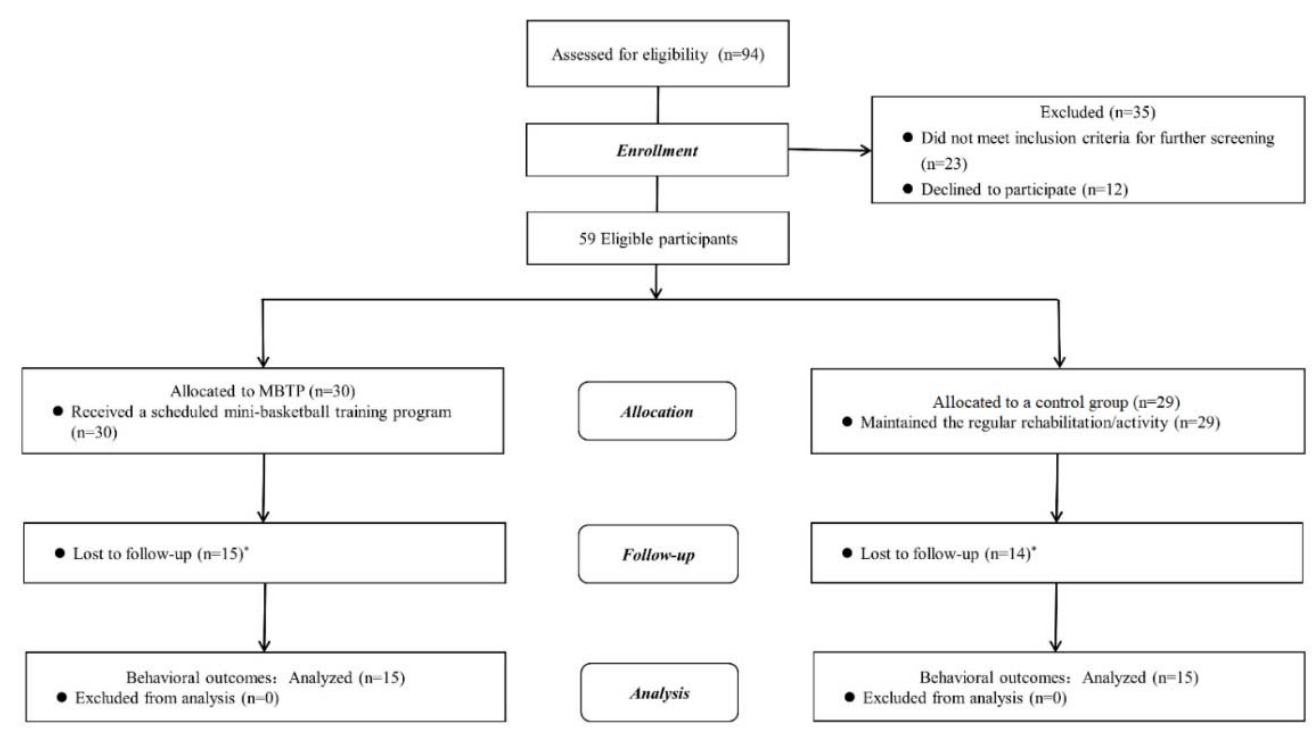

Figure 1. Participant flow characteristics.

* Twenty-nine children's parents did not finish the assessment in the post-test.

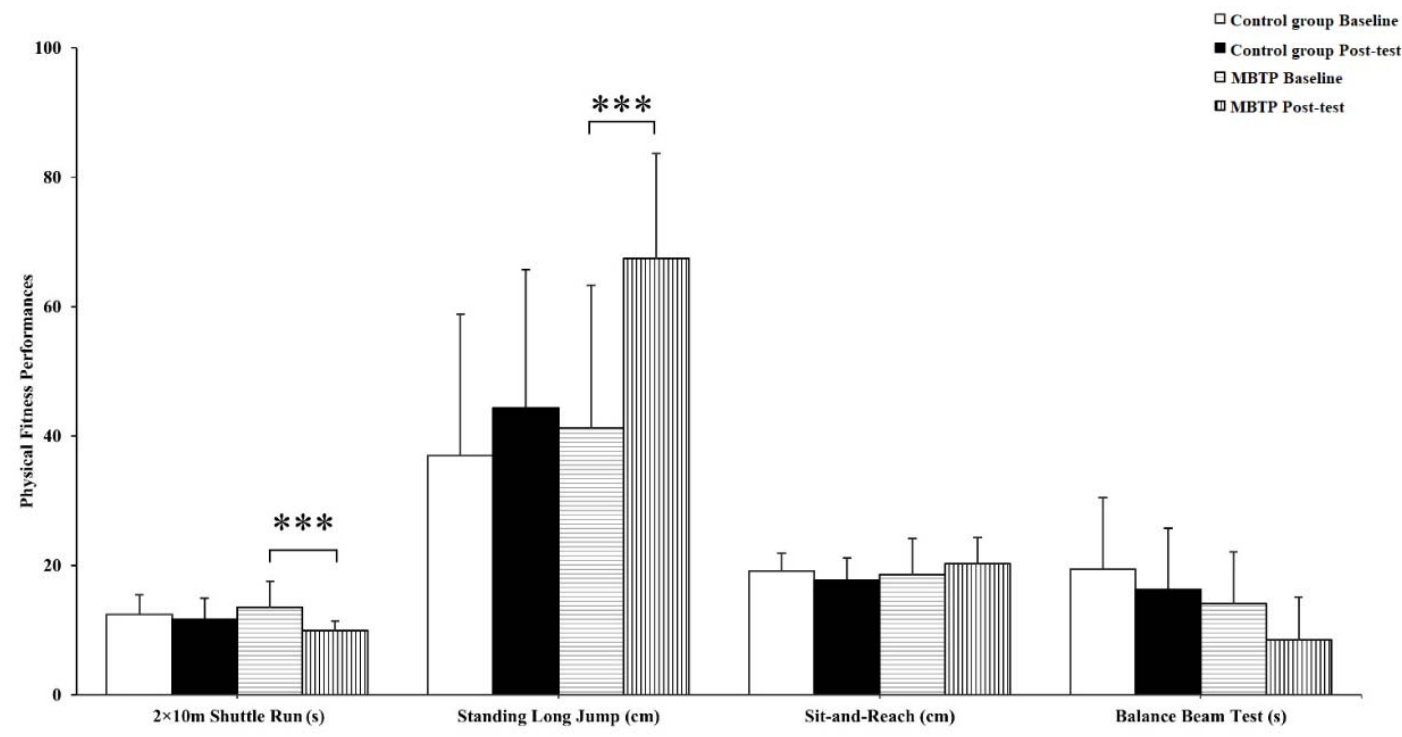

Figure 2. Performances for physical fitness assessment in particular time points (baseline vs. post-test) and group (control group vs. MBPT group).

${ }^{*}$ Indicates a significant difference between baseline and post-test $\left({ }^{* * *} p<.001\right)$. Error bars represent standard deviation (SD). 

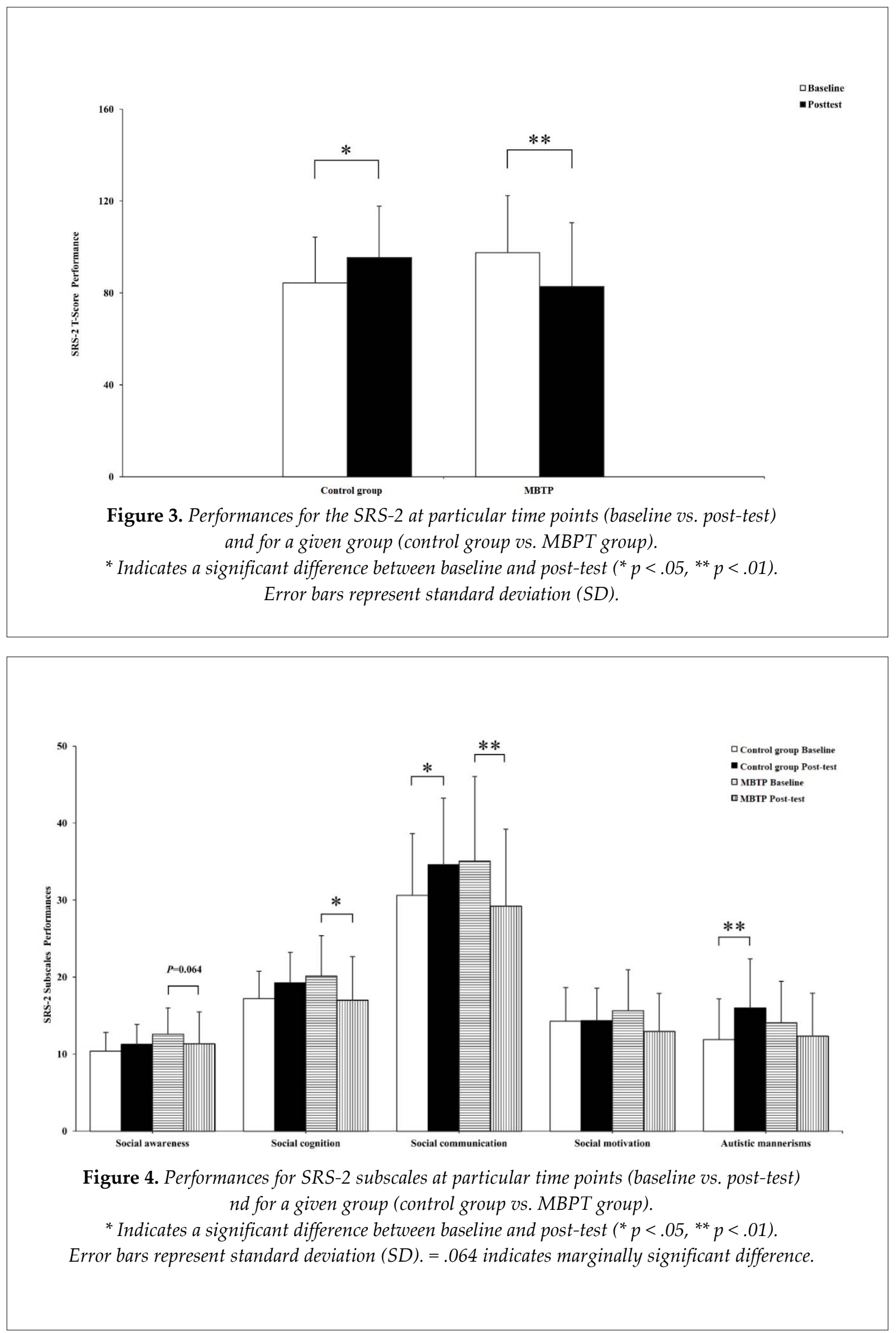
Table 1. Participant demographic characteristics $(M \pm S D)$.

\begin{tabular}{lcc}
\hline Variable & Control group & MBPT group \\
\hline $\mathrm{N}$ & 15 & 15 \\
Gender (boys/girls) & $14 / 1$ & $12 / 3$ \\
Age (years) & $4.56 \pm 0.84$ & $5.03 \pm 0.64$ \\
BMI (height/weight ${ }^{2}$ ) & $16.48 \pm 1.87$ & $15.70 \pm 1.33$ \\
CARS $^{\text {a }}$ & $38.73 \pm 3.78$ & $40.33 \pm 6.33$ \\
CSHQ $^{\text {b }}$ & $58.13 \pm 12.08$ & $56.13 \pm 5.02$ \\
CEBQ $^{c}$ & $54.27 \pm 19.97$ & $52.07 \pm 9.69$ \\
\hline
\end{tabular}

a CARS: Childhood Autism Rating Scale. ${ }^{b}$ CSHQ: Children's Sleep Habits Questionnaire. c CEBQ: Child Eating Behavior Questionnaire.

Table 2. Physical fitness and the SRS-2 performances ( $(M \pm S D)$.

\begin{tabular}{lcccc}
\hline \multirow{2}{*}{ Variable } & \multicolumn{2}{c}{ Control Group $(\mathrm{n}=15)$} & \multicolumn{2}{c}{ MBTP Group $(\mathrm{n}=15)$} \\
\cline { 2 - 5 } & Baseline & Posttest & Baseline & Posttest \\
\hline SRS-2 Performances (score) & & & & \\
SRS-2 T-score a & $84.33 \pm 19.93$ & $95.47 \pm 22.30$ & $97.53 \pm 24.97$ & $82.80 \pm 27.83$ \\
Social awareness & $10.40 \pm 2.41$ & $11.27 \pm 2.60$ & $12.60 \pm 3.397$ & $11.33 \pm 4.15$ \\
$\quad$ Social cognition & $17.20 \pm 3.55$ & $19.27 \pm 3.91$ & $20.13 \pm 5.26$ & $17.00 \pm 5.68$ \\
Social communication & $30.60 \pm 8.05$ & $34.60 \pm 8.65$ & $35.07 \pm 10.99$ & $29.20 \pm 10.00$ \\
Social motivation & $14.27 \pm 4.39$ & $14.33 \pm 4.23$ & $15.67 \pm 5.30$ & $12.93 \pm 4.94$ \\
Autistic mannerisms & $11.87 \pm 5.29$ & $16.00 \pm 6.37$ & $14.07 \pm 5.36$ & $12.33 \pm 5.58$ \\
Physical Fitness Performances & & & & \\
2 $\times$ 10 m shuttle run (s) & $12.40 \pm 3.06$ & $11.69 \pm 3.20$ & $13.56 \pm 3.97$ & $9.95 \pm 1.49$ \\
Standing long jump (cm) & $37.00 \pm 21.86$ & $44.33 \pm 21.37$ & $41.2 \pm 22.14$ & $67.47 \pm 16.16$ \\
Sit-and-reach (cm) & $19.17 \pm 2.73$ & $17.70 \pm 3.40$ & $18.58 \pm 5.58$ & $20.23 \pm 4.07$ \\
Balance beam test (s) & $19.40 \pm 11.02$ & $16.28 \pm 9.45$ & $14.12 \pm 7.97$ & $8.48 \pm 6.60$ \\
\hline
\end{tabular}

a The total score of the Social Responsiveness Scale Second Edition (SRS-2)

\section{Discussion}

The present study examined the effect of a $12-$ week MBTP on physical fitness and social communication in children with ASD. With the continued employment of fundamental movement skill training and playing games in the MBTP, the main findings revealed that the MBTP facilitated performance in speed-agility and increased muscle strength. Additionally, children with ASD in the MBTP group demonstrated improvements of specific social communication performances in social awareness, social cognition, social communication, and autistic mannerisms, whereas no influence was found in such performances in the control group. Although significant interaction effects were also revealed in other sub-indexes of physical fitness and social communication performances (i.e., sit-and-reach, balance beam test, and social motivation), no training effects were found, suggesting that minibasketball training sensitivity was limited in these aspects.

In the speed-agility and muscle strength tests 
increments in ASD children receiving the MBTP were found. The increments observed in physical fitness were consistent with previous studies which had demonstrated improvements in physical fitness after regular exercise. Minibasketball studies had provided supporting evidence regarding the considerable impact of the basic mini-basketball training on gross motor skills in children (Bagherly et al., 2012). A similar ASD study found that an exergame program could improve muscle strength and agility in children (Hilton et al., 2014). Since mini-basketball was the main content of the training program in the present study, it comprised plenty of practice in basketball with speed-agility and muscle strength e.g. basic mini-basketball skills as well as proper footwork and movement training.

Although the children's speed-agility and muscle strength improved through the MBTP, no improvements were observed for balance and flexibility. However, previous studies found that ASD can benefit from balance training programs to improve balance and postural control (Cheldavi et al., 2014). The results may be related to fewer balance and flexibility training sessions in the present program. For flexibility performance, Pan (2011) provided evidence that an aquatic program could promote flexibility for ASD children. This finding, however, is inconsistent with the present results. The results indicated that the sit-and-reach test produced a 'ceiling effect' at baseline, resulting in no significant improvement after the MBTP. These explanations are admittedly speculative, and future research is required before any definitive conclusions may be drawn.

A part of the results supports our hypothesis that a 12-week MBTP is beneficial to ASD children's social communication. The findings were similar to existing studies that demonstrated that swimming, aerobics (Pan, 2010), horseback riding (Bass et al., 2009; Gabriels et al., 2012), Karate training (Movahedi et al., 2013) and leisure activity programs (Garcia-Villamisar and Dattilo, 2011) had positive effects on social communication with ASD children. In the present study, improvements observed in social communication may be attributed to repeating teachers' movements during training sessions, which promoted children's social skills when learning basketball motor skills. One study reported that imitation treatment was an effective approach to improve social communication in ASD children (Ferraioli and Harris, 2011). There is also another possibility. To accomplish minibasketball training, the inevitable communication behaviors with their partners or parent compelled children to yield social behaviors. Similar discoveries were found in Karate training, where social communication of children with ASD benefited from watching their peers practice (Movahedi et al., 2013). Moreover, basic social etiquettes, such as greeting peers, greeting parents or teachers and waiting in line during the basketball training sessions may also facilitate ASD children yield social behavior (Movahedi et al., 2013). More importantly, the MBTP session was presented to children in the form of a collective class, each training session was designed to induce and reinforce social communication behavior. Thus, our results demonstrated that the MBTP promoted social communication in children with ASD.

Although significant results were found in social awareness, social cognition, social communication, and autistic mannerisms, the effects on social motivation were not obvious, which was inconsistent with previous studies. A recent study showed that children with ASD exposed to therapeutic horseback riding exhibited greater social motivation (Bass et al., 2009). However, the MBTP was primarily directed towards sensory stimulation, emphasizing fine motor and perceptual skills, but not towards motivation training. In addition, the 12-week training period of the MBTP should last for a longer training period than in the current study, because of obvious social motivation changes. This is a plausible hypothesis as the other subscales were significant. Therefore, the followup observation is necessary to discover whether the MBTP gains are transient or persist if training continues.

Social and behavioral deficits among children with ASD make interaction with peers difficult, and such physical and social constraints had shown to reduce the physical fitness level in children with ASD (Pan and Frey, 2006). Meanwhile, the sedentary lifestyle not only affects a child's general health and family dynamics, but may further isolate and deprive the child of social adaptive functions and skills (Yu et al., 2018). Our 
findings showed that the MBTP could improve physical fitness as well as social communication in preschool children with ASD. These findings also indicated that the MBTP may alter physical fitness in ASD leading to social communication gains. The speculation is only discussed in the theoretical perspective as there is no strong evidence to support this speculation. Future studies are warranted to explain this aspect.

The consistent improvement in social communication in the participants of the MBTP group in our study may also be explained from the neuroplasticity point of view. Extensive neuropsychological and neuroimaging evidence indicated that brain abnormalities in ASD were associated with social communication impairment (Fishman et al., 2015; Nickl-Jockschat et al., 2015; Shen et al., 2016). The latest study demonstrated that favorable environmental stimulation (music intervention) altered functional brain activity in ASD leading to social communication gains (Sharda et al., 2018). Physical exercise, as external stimulation, has also been shown to influence the plasticity of children's brain development (Chen et al., 2016; Davis et al., 2011; Krafft et al., 2014a, 2014b). Therefore, we speculate that a 12-week MBTP might promote the enhancement of brain plasticity, which may improve the social communication of children with ASD. Since there was no research on the neural mechanism of sports to improve social communication in children with ASD, the above speculation is only discussed from the theoretical point of view. Although we did not collect any neuroimaging data in the current study, the neural mechanism should be further explored.
To our knowledge, the present study was the first to focus on the effect of an exercise intervention on physical fitness and social communication in a population of preschool children with ASD; it may mean that exercise plays an important role in the adjuvant treatment of ASD behavioral problems and motor abilities. In addition, our MBTP was a multidimensional training program that differed from previous training programs and included basic movement skill training and social behavior reinforcement. Furthermore, the present study had controlled the confounding variables as much as possible. Therefore, reliable training gains emerged in ASD physical fitness and social communication. However, there are still numerous aspects to be improved. Although the SRS-2 was an authoritative scale to assess children's social communication impairment, the parent-reported measure was certainly subjective. However, no better assessment tool to evaluate preschool ASD children's social communication impairment exists. Thus, generalization in terms of our program's effects on social communication should proceed cautiously.

\section{Conclusion}

In summary, our study provides initial evidence that 12 weeks of the MBTP exerted a positive effect on physical fitness and social communication in preschool children with ASD. These enhancements further elucidate the potential application of mini-basketball training as a complementary intervention for rehabilitating social communication impairment and increasing physical fitness in preschool children with ASD.

\section{Acknowledgments}

The authors would like to express their gratitude to all the children who participated in this study, teachers and parents of children for their support, and research assistants who helped with data collection and other contributions. This research was supported by grants from the National Natural Science Foundation of China (31771243), the Fok Ying Tong Education Foundation (141113) to Ai-Guo Chen and Yangzhou University Science and Technology Foundation(X20180248) to Ke-Long Cai.

\section{References}

American Psychiatric Association. Diagnostic and statistical manual of mental disorders (DSM-5®). Washington: American Psychiatric Association; 2013

Bagherly J, Ghasemi A. The Compensatory Impact of Mini-Basketball Skills on the Progress of Fundamental Movements in Children. Procedia Soc Behav Sci, 2012; 46: 5206-5210

Baio J, Wiggins L, Christensen DL, Maenner MJ, Daniels J, Warren Z, Kurzius-Spencer M, Zahorodny W, 
Robinson Rosenberg C, White T, Durkin MS, Imm P, Nikolaou L, Yeargin-Allsopp M, Lee LC, Harrington R, Lopez M, Fitzgerald RT, Hewitt A, Pettygrove S, Constantino JN, Vehorn A, Shenouda J, Hall-Lande J, Van Naarden Braun K, Dowling NF. Prevalence of Autism Spectrum Disorder Among Children Aged 8 Years - Autism and Developmental Disabilities Monitoring Network, 11 Sites, United States, 2014. MMWR Surveill Summ, 2018; 67: 1-23

Bass MM, Duchowny CA, Llabre MM. The Effect of Therapeutic Horseback Riding on Social Functioning in Children with Autism. J Autism Dev Disord, 2009; 39: 1261-1267

Bedford R, Pickles A, Lord C. Early gross motor skills predict the subsequent development of language in children with an autism spectrum disorder. Autism Res, 2016; 9: 993-1001

Bremer E, Crozier M, Lloyd M. A systematic review of the behavioral outcomes following exercise interventions for children and youth with an autism spectrum disorder. Autism, 2016; 20: 899-915

Cheldavi H, Shakerian S, Shetab Boshehri SN, Zarghami M. The effects of balance training intervention on postural control of children with autism spectrum disorder: Role of sensory information. Res Autism Spectr Disord, 2014; 8: 8-14

Chen AG, Zhu LN, Yan J, Yin HC. Neural Basis of Working Memory Enhancement after Acute Aerobic Exercise: fMRI Study of Preadolescent Children. Front Psychol, 2016; 7: 1804

Constantino J, Gruber CP. The social responsiveness scale (SRS) manual: Los Angeles: Western Psychological Services; 2005

Davis CL, Tomporowski PD, McDowell JE, Austin BP, Miller PH, Yanasak NE, Allison JD, Naglieri JA. Exercise Improves Executive Function and Achievement and Alters Brain Activation in Overweight Children: A Randomized, Controlled Trial. Health Psychol, 2011; 30: 91-98

Erdfelder E, Faul F, Buchner A, Lang AG. Statistical power analyses using G*Power 3.1: Tests for correlation and regression analyses. Behavior Behav Res Methods, 2009; 41: 1149-1160

Ferraioli SJ, Harris SL. Treatments to Increase Social Awareness and Social Skills. Evidence-based practices and treatments for children with autism; New York; Springer:171-196; 2010

Fishman I, Datko M, Cabrera Y, Carper RA, Müller RA. Reduced integration and differentiation of the imitation network in autism: A combined functional connectivity magnetic resonance imaging and diffusion-weighted imaging study. Ann Neurol, 2015; 78: 958-969

Gabriels RL, Agnew JA, Holt KD, Shoffner A, Zhaoxing P, Ruzzano S, Clayton, GH, Mesibov G. Pilot study measuring the effects of therapeutic horseback riding on school-age children and adolescents with autism spectrum disorders. Res Autism Spectr Disord, 2012; 6: 578-588

Garcia-Villamisar D, Dattilo J. Social and clinical effects of a leisure program on adults with autism spectrum disorder. Res Autism Spectr Disord, 2011; 5: 246-253

General Administration of Sport of China. China's measurements manual of physical fitness: People's Sports Publishing House of China; 2003

Goodlin-Jones BL, Sitnick SL, Tang K, Liu J, Anders TF. The Children's Sleep Habits Questionnaire in toddlers and preschool children. J Dev Behav Pediatr, 2008; 29: 82-88

Hilton CL, Cumpata K, Klohr C, Gaetke S, Artner A, Johnson H, Dobbs S. Effects of exergaming on executive function and motor skills in children with autism spectrum disorder: A pilot study. Am J Occup Ther, 2014; 68: 57-65

Krafft C E, Schaeffer DJ, Schwarz NF, Chi L, Weinberger AL, Pierce JE, Rodrigue AL, Allison JD, Yanasak NE, Liu T, Davis CL, McDowell JE. Improved front oparietal white matter integrity in overweight children is associated with attendance at an after-school exercise program. Dev Neurosci, 2014a; 36: 1-9

Krafft CE, Schwarz NF, Chi L, Weinberger AL, Schaeffer DJ, Pierce JE, Rodrigue AL, Yanasak NE, Miller PH, Tomporowski PD, Davis CL, McDowell JE. An 8-month randomized controlled exercise trial alters brain activation during cognitive tasks in overweight children. Obesity, 2014b; 22: 232-242

Lambert JM, Copeland BA, Karp EL, Finley CI, Houchins-Juarez NJ, Ledford JR. Chaining Functional Basketball Sequences (with Embedded Conditional Discriminations) in an Adolescent with Autism. Behav Anal Pract, 2016; 9: 199-210

Lang R, Koegel LK, Ashbaugh K, Regester A, Ence W, Smith W. Physical exercise and individuals with autism spectrum disorders: A systematic review. Res Autism Spectr Disord, 2010; 4: 565-576

Movahedi A, Bahrami F, Marandi SM, Abedi A. Improvement in social dysfunction of children with autism spectrum disorder following long term Kata techniques training. Res Autism Spectr Disord, 2013; 7: 
1054-1061

Müller RA, Fishman I. Brain Connectivity and Neuroimaging of Social Networks in Autism. Trends Cogn Sci, 2018; 22: 1103-1116

Must A, Phillips SM, Curtin C, Anderson SE, Maslin M, Lividini K, Bandini LG. Comparison of sedentary behaviors between children with autism spectrum disorders and typically developing children. Autism, 2014; 18: 376-384

Nickl-Jockschat T, Rottschy C, Thommes J, Schneider F, Laird AR, Fox PT, Eickhoff SB. Brain Struct Funct, 2015; 220: 2355-2371

Owens JA, Spirito A, McGuinn M. The Children's Sleep Habits Questionnaire (CSHQ): Psychometric properties of a survey instrument for school-aged children. Sleep, 2000; 23: 1043-1051

Pan CY. Effects of water exercise swimming program on aquatic skills and social behaviors in children with autism spectrum disorders. Autism, 2010; 14: 9-28

Pan CY. The efficacy of an aquatic program on physical fitness and aquatic skills in children with and without autism spectrum disorders. Res Autism Spectr Disord, 2011; 5: 657-665

Pan CY, Frey GC. Physical activity patterns in youth with autism spectrum disorders. J Autism Dev Disord, 2006; 36: 597-606

Petrus C, Adamson SR, Block L, Einarson SJ, Sharifnejad M, Harris SR. Effects of exercise interventions on stereotypic behaviours in children with autism spectrum disorder. Physiother Can, 2008; 60: 134-145

Pitetti KH, Rendoff AD, Grover T, Beets MW. The efficacy of a 9-month treadmill walking program on the exercise capacity and weight reduction for adolescents with severe autism. J Autism Dev Disord, 2007; 37: 997-1006

Rice CE, Lee LC. Expanding the global reach of research in autism. Autism, 2017; 21: 515-517

Schopler E, Reichler RJ, DeVellis RF, Daly K. Toward objective classification of childhood autism: Childhood Autism Rating Scale (CARS). J Autism Dev Disord, 1980; 10: 91-103

Sharda M, Tuerk C, Chowdhury R, Jamey K, Foster N, Custo-Blanch M, Tan M, Nadig A, Hyde K. Music improves social communication and auditory-motor connectivity in children with autism. Transl Psychiatry, 2018; 8: 1-13

Shen MD, Li DD, Keown CL, Lee A, Johnson RT, Angkustsiri K, Rogers SJ, Müller RA, Amaral DG, Nordahl CW. Functional Connectivity of the Amygdala Is Disrupted in Preschool-Aged Children With Autism Spectrum Disorder. J Am Acad Child Adolesc Psychiatry, 2016; 55: 817-824.

Sorensen C, Zarrett N. Benefits of Physical Activity for Adolescents with Autism Spectrum Disorders: A Comprehensive Review. J Autism Dev Disord, 2014; 1: 344-353

Sowa M, Meulenbroek R. Effects of physical exercise on Autism Spectrum Disorders: A meta-analysis. Res Autism Spectr Disord, 2012; 6: 46-57

Todd T, Reid G. Increasing Physical Activity in Individuals With Autism. Focus Autism Other Dev, 2006; 21: 167-176

Tyler K, MacDonald M, Menear K. Physical activity and physical fitness of school-aged children and youth with autism spectrum disorders. Autism Res Treat, 2014: 312163

Wang L, Wang F. Effect of early intervention on children with autism and its influencing factors. J Psychiatry, 2014; 27: 350-352

Wardle J, Guthrie CA, Sanderson S, Rapoport L. Development of the children's eating behaviour questionnaire. J Child Psychol Psychiatry, 2001; 42: 963-970

Yu CCW, Wong SWL, Lo FSF, So RCH, Chan DFY. Study protocol: a randomized controlled trial study on the effect of a game-based exercise training program on promoting physical fitness and mental health in children with autism spectrum disorder. BMC Psychiatry, 2018; 18: 1-10

Zhao LX, Fan JY. Symptoms and pathogenic mechanism of social communication and interaction defect in autism spectrum disorder children. Chinese J Child Health Care, 2017; 25: 585-587

Zhu Y, Xu C, Wan Q, Guo LY. Effects of adapted physical exercise intervention on visual working memory in children with autism spectrum disorder. China Sport Sci Tech, 2017; 53: 55-62

\section{Corresponding author:}

Ai-Guo Chen, College of Physical Education; Room ES509, Wenti Building of Yangzijin Campus,196 West Huayang Road,Yangzhou, Jiangsu, China; Code:225127; E-mail: agchen@yzu.edu.cn 